\title{
The Systemic Management of Advanced Melanoma in 2016
}

\author{
Markus V. Heppt Cecilia Dietrich Saskia A. Graf Thomas Ruzicka Julia K. Tietze \\ Carola Berking
}

Department of Dermatology and Allergy, Munich University Hospital (LMU), Munich, Germany

\section{Keywords}

Melanoma · Nivolumab · Pembrolizumab · BRAF · MEK

\section{Summary}

Melanoma is a common type of skin cancer with a high propensity to metastasize. Tyrosine kinase inhibitors targeting the mitogen-activated protein kinase (MAPK) pathway and immune checkpoint blockade have recently revolutionized the management of unresectable and metastatic disease. However, acquired resistance and primary non-response to therapy require novel treatment strategies and combinations. The purpose of this review is to provide a brief and up-to-date overview on the clinical management and current trial landscape in melanoma. We summarize the most pertinent studies on BRAF/MEK inhibitors and blockade of cytotoxic T-lymphocyte-associated protein 4 (CTLA-4) and programmed cell death protein 1 (PD-1). Although most agents show robust antitumor efficacy as single agents, further improvements have been achieved by the combination of both approved and developing drugs. We discuss ongoing trials and evaluate future approaches that may provide additional efficacy with less toxicity.

(C) 2016 S. Karger GmbH, Freiburg

\section{Introduction}

In the last few years, the therapy for advanced melanoma has been revolutionized by introducing a panel of novel local and systemic treatment approaches. In particular, targeted BRAF mutation status-based therapies with tyrosine kinase inhibitors and immune therapy with ipilimumab or programmed cell death protein 1
(PD-1) inhibitors are now the pillars of a modern and effective treatment (figs. 1 and 2). They have replaced the use of chemotherapy with dacarbazine and other cytotoxic agents in clinical practice. Further improvements could well be achieved by combining therapies that are presently available. In this review article, we summarize the current trials and the state-of-the-art systemic therapies for advanced melanoma.

\section{Targeted Therapy with Kinase Inhibitors (BRAF and MEK Inhibitors)}

If the oncogene BRAF harbors activating mutations such as Val600Glu (V600E) or Val600Lys (V600K), targeted therapy with tyrosine kinase-inhibiting substances should be considered in the first- or second-line treatment. Several phase III studies indicate, in a very impressive way, that an upfront combination therapy with inhibitors to BRAF (BRAFi) and MEK (MEKi) is superior to BRAFi monotherapy alone (table 1).

\section{Dabrafenib and Trametinib}

The double-blind, randomized phase III study COMBI-d compared dabrafenib and trametinib to dabrafenib alone. In a primary analysis after a median follow-up of 9 months, the combination was superior to monotherapy with a progression-free survival (PFS) of 9.3 and 8.8 months, respectively, and a hazard ratio (HR) for progression or death of 0.75 (95\% confidence interval (CI) $0.57-0.99$ ). The objective response rate (ORR) lay at $67 \%$ for the combination and at $51 \%$ for the monotherapy cohort [1]. A final report on overall survival (OS) was performed when $70 \%$ of the intention-to-treat population had died [2]. The median OS was 25.1 months in the dabrafenib plus trametinib group versus 18.7 months in the dabrafenib only group (HR 0.71 ; 95\% CI $0.55-$ $0.92)$. In the group treated with the combination, $74 \%$ and $51 \%$ of the patients survived the first and second year under observation.

\section{KARGER}

(c) 2016 S. Karger GmbH, Freiburg

Fax +497614520714 


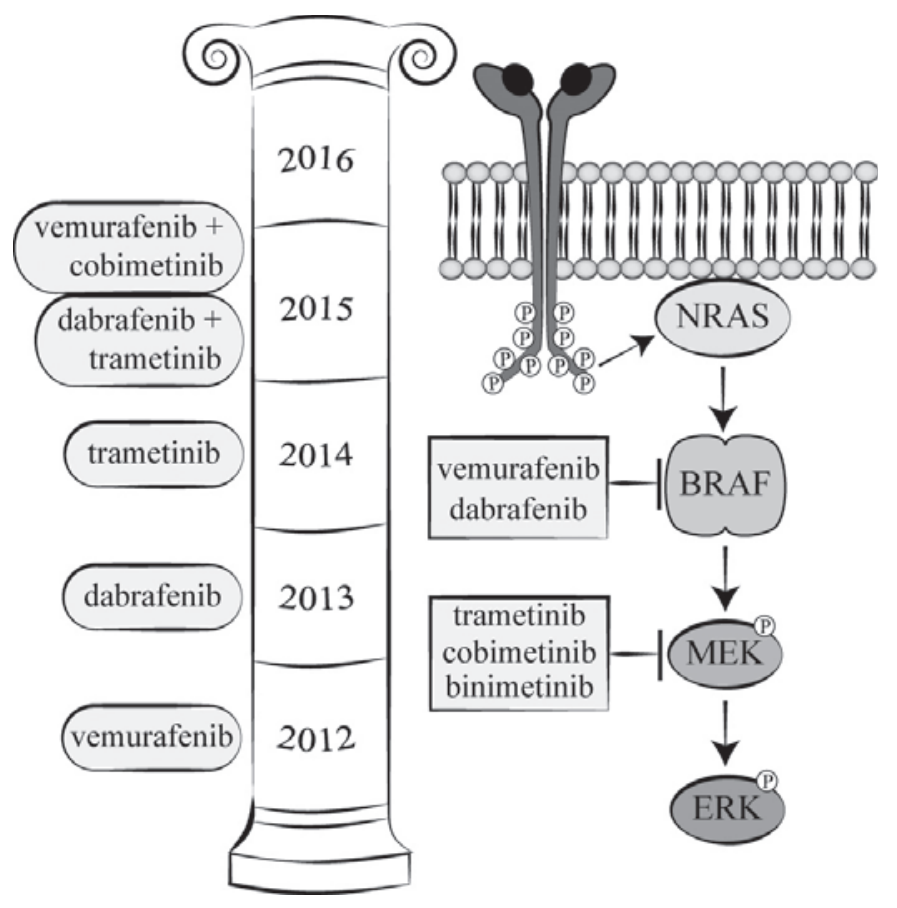

Fig. 1. The mitogen-activated protein kinase (MAPK)-signaling pathway can be induced by activating mutations of receptor tyrosine kinases and mediated through the small guanosine triphosphate (GTP)ase NRAS, the serine/threonine kinase BRAF and the MAPK kinases MEK and ERK. Specific BRAF inactivation can be achieved with the inhibitors vemurafenib and dabrafenib, while cobimetinib, trametinib, and binimetinib block MEK kinase activity. These inhibitors have been approved in single and combination therapy by the European Medicines Agency (EMA) in the years as depicted in the pillar.

The PFS in the combination arm increased further to 11.0 months (HR 0.67; 95\% CI 0.53-0.84). The response rates were 69\% and $53 \%$, respectively [2]. In addition to objective clinical benefits such as delayed progression or longer OS, patients receiving the combination showed a better health-related quality of life along with significant functional and pain improvements compared with patients receiving monotherapy with dabrafenib. However, some dimensions of the questionnaires such as nausea and vomiting or diarrhea showed a trend of being favorable with dabrafenib only [3]. Recently, a 3-year analysis was presented (with a cut-off date for data of 15 February 2016), revealing a PFS of $22 \%$ (dabrafenib plus trametinib) versus $12 \%$ (dabrafenib only) and OS of $44 \%$ versus $32 \%$. The outcome was best in patients with normal serum lactate dehydrogenase (LDH) and less than 3 affected organ systems at baseline [4]. These data provide the so far longest follow-up on OS in patients receiving BRAFi and MEKi in a phase III trial setting.

The open-label, randomized phase III trial COMBI-v compared dabrafenib and trametinib to vemurafenib as first-line therapy in 704 patients with metastatic melanoma with OS as primary endpoint [5]. The study was prematurely stopped for efficacy based on a positive interim analysis. Median OS was 17.2 months for the vemurafenib only group and was not reached by patients who were treated with the combination. The survival benefit was evident in all specified subgroups except for patients with an Eastern Cooperative Oncology Group (ECOG) score of 1 at baseline. Median

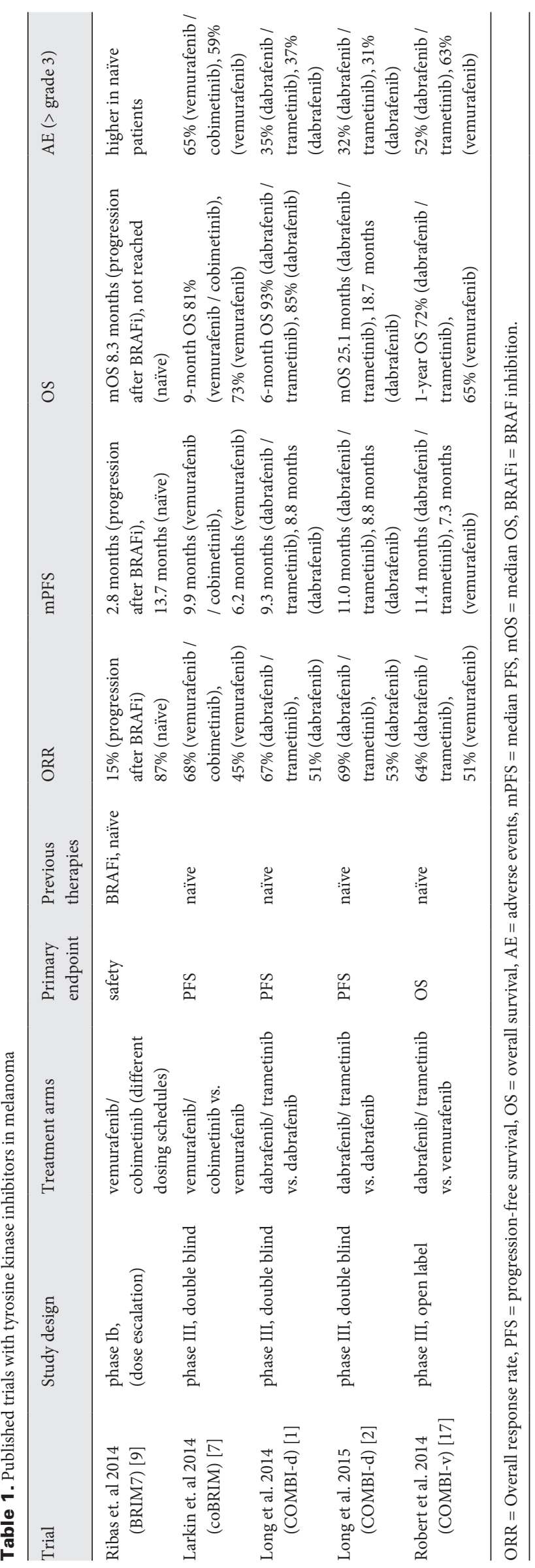

Heppt/Dietrich/Graf/Ruzicka/Tietze/Berking 
Fig. 2. Blocking cytotoxic T-lymphocyte-associated protein 4 (CTLA-4) or programmed cell death protein 1 (PD-1) signaling in tumor immunotherapy. T cells recognize antigens that are presented by dendritic cells (DC) or melanoma cells through the major histocompatibility complex (MHC) with their T-cell receptors (TCR). Several signals regulate T-cell activity. They can be activated through costimulatory $\mathrm{B} 7$ molecules binding to $\mathrm{CD} 28$, while binding of B7 to CTLA-4 triggers inhibitory signals during the priming phase in lymph nodes. Binding of programmed death ligand 1 (PD-L1) on melanoma cells to PD-1 on T cells results in negative regulation during the effector phase in the peripheral tissue. Thus, T-cell activation can be achieved by antibody-mediated blocking of

CTLA-4 with ipilimumab or PD-1 with nivolumab or pembrolizumab. Years in which these antibodies have been approved for melanoma therapy by the EMA are depicted in the pillar.

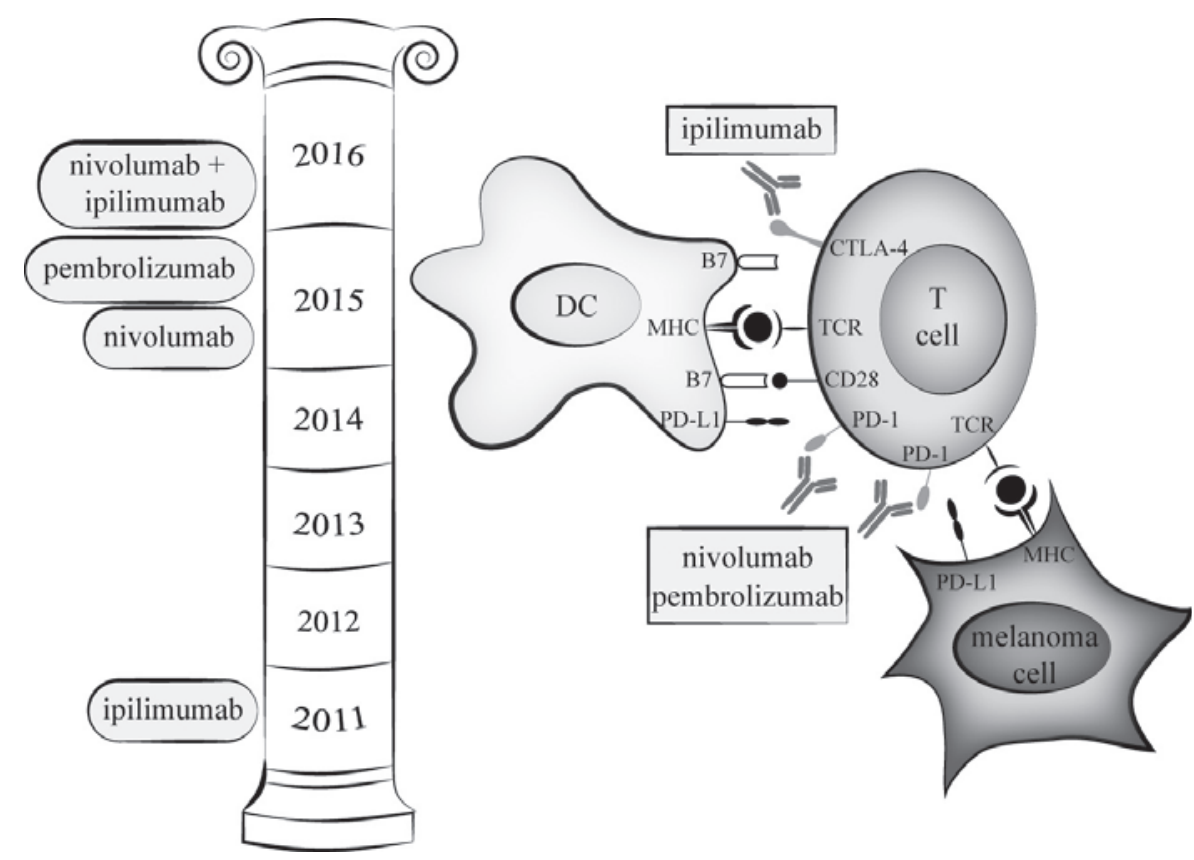

PFS was 11.4 versus 7.3 months (HR 0.56; 95\% CI 0.46-0.69) with best overall response rates of $64 \%$ and $51 \%$, respectively [5]. Analogous to COMBI-d, scores for global health and most functional and disease symptoms showed significant improvements in favor of the combination, further underlining that the upfront combination of BRAFi and MEKi is the standard of care for patients with activating BRAFV600 mutations [6].

\section{Vemurafenib and Cobimetinib}

The combination of vemurafenib and cobimetinib was approved by the European Medicines Agency (EMA) in December 2015 based on the results of the pivotal coBRIM trial. Patients who had not previously been treated for unresectable or metastatic melanoma received vemurafenib and cobimetinib or vemurafenib plus placebo with investigator-assessed PFS as primary endpoint [7]. The rate of complete or partial responses was $68 \%$ for the combination and $45 \%$ for the control group. After 9 months of follow-up, survival rates were $81 \%$ and $73 \%$, respectively. The median PFS was 9.9 months in the combination group and 6.2 months for vemurafenib monotherapy [7]. After a median follow-up of 14.2 months, PFS further increased to 12.3 and 7.2 months. Vemurafenib plus cobimetinib showed a better ORR (70\%) compared to that for vemurafenib plus placebo (50\%). Of note, $16 \%$ of patients in the combination arm showed complete response. The duration of the response was 13.0 and 9.2 months, respectively. The treatment-related benefits were consistent in every assessed patient subgroup, including patients with BRAFV600E and BRAFV600K mutations [8]. The survival data for the coBRIM were recently updated after a median follow-up of 18.5 months. After 1 year, $75 \%$ of patients treated with the combination were alive. The 2-year survival rate was still $48 \%$. Median OS amounted to 22.3 months for the combination as opposed to 17.4 months for vemurafenib only (HR 0.70; 95\%CI 0.55-0.90). Even in patients with increased LDH with an unfavorable prognosis, the survival time was significantly better. On the other hand, for the expression of other molecular tumor features, such as the proliferation marker Ki-67 or the tumor-suppressors PTEN, no difference was detected with respect to the OS rate.

Similar results were reported for BRIM7 after an extended follow-up. BRIM7 was initially designed as a dose-escalating phase Ib trial, which formed the basis for the development of the vemurafenib and cobimetinib combination regimen [9]. Included study patients had either previously progressed on vemurafenib or were BRAFi naïve. A striking confirmed response rate of $87 \%$ was observed in the latter group with 4 more patients attaining a complete response at cycles 16-25. The median PFS was left unchanged at 13.8 months and the median OS was reached at 28.5 months. After 2 years of follow-up, $61 \%$ of patients receiving vemurafenib and cobimetinib were still alive [10].

\section{Binimetinib (MEK162)}

Activating mutations of the oncogene NRAS are present in approximately $20 \%$ of patients with melanoma. Some studies proposed that the NRAS mutation status is an independent predictor of short survival in stage IV disease [11]. Thus, NRAS-mutated patients are at high risk for disease progression and represent a population with unmet clinical need, in particular after immune checkpoint blockade has failed. Preclinical studies have shown that NRAS-mutated melanoma is sensitive to MEK inhibition [12, 13]. Binimetinib (MEK162), an oral inhibitor of both MEK1 and MEK2, was the first targeted therapy to show antitumor activity in patients with NRAS-mutated melanoma in a phase II trial [14]. This was the rationale for the NEMO trial to test binimetinib in a phase III setting in NRASQ61-mutated melanoma. Patients were randomized at a 2:1 ratio to either binimetinib or dacarbazine. Binimetinib significantly improved PFS over dacarbazine with a me- 
dian PFS of 2.8 versus 1.5 months (HR 0.62; 95\% CI $0.47-0.80$ ). The response rates in the intention-to-treat population were $15 \%$ and $7 \%$, respectively. The median OS was not significantly improved and lay at 11.0 months [4].

\section{Safety and Tolerability}

Although in the coBRIM study incidences of severe adverse events in the combination therapy were slightly elevated, other studies and the clinical practice have shown that the safety and the tolerability of both combinations are good. Cutaneous side effects such as the development of palmoplantar hyperkeratosis, squamous cell carcinoma, or hair loss, which frequently occur under BRAFi monotherapy, are found less frequently with the combination due to simultaneous inhibition of $\operatorname{MEK}[2,5]$. However, nausea, vomiting and diarrhea occur more frequently in the combination regimens. One of the most frequent adverse events of dabrafenib plus trametinib is pyrexia, which has been observed in more than $50 \%$ of all cases $[1,5]$. On the other hand, with vemurafenib plus cobimetinib, photosensitivity has been observed in $20 \%$ of the cases [7]. Moreover, an important effect of MEKi is the reduction of the left ventricle ejection fraction, even to the extent of a clinically manifested heart insufficiency, as well as the development of a central serous retinopathy. Regular cardiac examinations with electrocardiography and trans-thorax echocardiograms should be performed every 3 months as well as an examination of the retina with optical coherence tomography prior to treatment.

\section{Immune checkpoint blockade}

\section{Ipilimumab Monotherapy}

The antibody ipilimumab, which is directed against cytotoxic $\mathrm{T}$ lymphocyte-associated protein 4 (CTLA-4), is administered intravenously for 4 cycles at 3-weekly intervals, in contrast to the PD-1 inhibitors, which are applied as continuous therapy. Pooled data from several phase 11 and phase 111 trials have recently been collected and analyzed, revealing a median survival time of 11.4 months for ipilimumab monotherapy [15]. Interestingly, the survival curves reach a plateau after 3 years and appear stable even 10 years post treatment. According to the studies that have been published thus far, the response rates of ipilimumab as monotherapy are lower than those with PD-1 inhibitors, and the rate of adverse events is presumably higher. Events such as autoimmune colitis and hypophysitis may require hospitalization and result in irreversible hormone deficiency. Thus, despite its proven long-term efficacy and the limited number of applications, the future of ipilimumab as monotherapy in melanoma is uncertain.

\section{Pembrolizumab Monotherapy}

Pembrolizumab is administered intravenously at $2 \mathrm{mg} / \mathrm{kg}$ body weight every 3 weeks and can be administered continuously over a period of at least 1-2 years if the response is favorable and the drug well tolerated. However, the optimal treatment duration has not been identified to date [16]. It was first evaluated in the large phase
I KEYNOTE-001 study. After a median follow-up duration of 18 months, the response rate was $34 \%$ and the median OS 25.9 months. The responses were stable and maintained in $81 \%$ of patients, suggesting a high antitumor efficacy in the early stages of drug development [17]. The KEYNOTE-002 study compared 2 dosage schemes of pembrolizumab in comparison to investigatorchoice chemotherapy (ICC) in patients who were refractory to ipilimumab or to prior BRAFi. Both dosages of pembrolizumab, $2 \mathrm{mg} / \mathrm{kg}$ and $10 \mathrm{mg} / \mathrm{kg}$ body weight, were clearly superior to ICC with respect to PFS and tolerability, providing the basis for accelerated approval in advanced melanoma [18]. Pembrolizumab was ultimately compared to ipilimumab in the phase III trial KEYNOTE-006. It was administered at $10 \mathrm{mg} / \mathrm{kg}$ body weight in 2 distinct cohorts every 2 and 3 weeks, respectively. The efficacy was similar in both of these groups and significantly higher over ipilimumab with an estimated 6-month PFS of $46-47 \%$ compared to $27 \%$ (HR 0.58; 95\% CI 0.46-0.72). The response rates were $34 \%$ (2-week cycle) and 33\% (3-week cycle) in comparison to $12 \%$ for patients with ipilimumab monotherapy [19]. Regarding safety, pembrolizumab was better tolerated than both chemotherapy (shown in KEYNOTE-002) and ipilimumab (shown in KEYNOTE-006). In 3-7\% of patients treatment was discontinued due to severe treatment-related adverse events. The most common events observed with pembrolizumab are fatigue, diarrhea, rash, and pruritus. Immune-related side effects most commonly are hyper- and hypothyroidism, colitis, hepatitis, hypophysitis, and pneumonitis, although virtually any organ system can be affected [19-21].

\section{Nivolumab Monotherapy}

Nivolumab is administered intravenously at $3 \mathrm{mg} / \mathrm{kg}$ body weight every 2 weeks. The phase III study CheckMate 037 evaluated nivolumab in comparison to ICC as second- or later-line treatment in patients who were refractory to ipilimumab or BRAFi. Confirmed objective responses were reported in $31.7 \%$ and $10.6 \%$, respectively [22]. These results were further corroborated in previously untreated patients without a BRAF mutation in the trial CheckMate 066, where nivolumab was compared to dacarbazine as first-line approach. Patients in the nivolumab arm showed a response rate of $40.0 \%$ versus $13.9 \%$ in the dacarbazine arm. The median PFS was 5.1 and 2.2 months, respectively (HR 0.43; 95\% CI $0.34-0.56)$. After 1 year of follow-up, the survival rates were $73 \%$ and $42 \%$, respectively (HR $0.42 ; 99.8 \%$ CI $0.25-0.73$ ). This survival benefit was evident in all pre-specified subgroups including those that were defined by expression of the programmed death ligand 1 (PD-L1), indicating that nivolumab is effective irrespective of PD-L1 expression levels [23]. Recently, updated data have been presented after an observational time of 18.5 months post therapy. After this period of time, the median OS rate had not yet been reached in the nivolumab cohort, whereas it was 11.2 months for dacarbazine (HR 0.43; 95\% CI 0.33-0.57). The median PFS further improved to 5.4 months for nivolumab and remained at 2.2 months with chemotherapy (HR 0.42; 95\% CI 0.32-0.53). The objective response rates were $42.9 \%$ and $14.4 \%$, respectively. The 


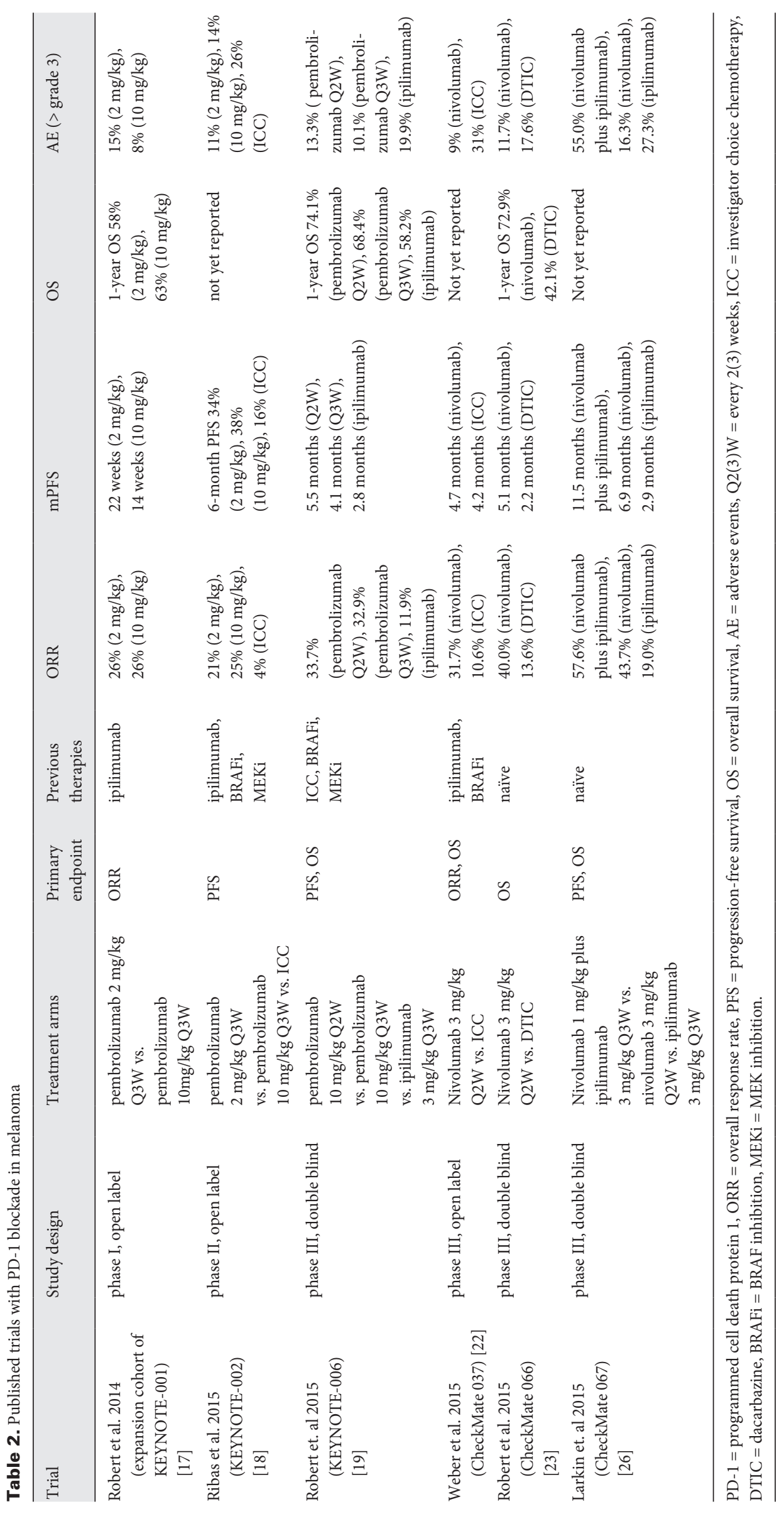


2 -year OS for patients who received nivolumab was $68.3 \%$ for patients with $\geq 5 \%$ PD-L1 expression and 54.2\% for those with $<5 \%$ PD-L1 expression. Treatment-related severe adverse events were observed in $10-13 \%$ of patients and comprised fatigue, pruritus, nausea, diarrhea, and rash. Frequent laboratory abnormalities under nivolumab treatment are increased levels of serum lipase and alanine aminotransferase [23].

\section{Ipilimumab plus Nivolumab}

Several studies provide strong evidence that a primary combination of CTLA-4 and PD-1/PD-L1 blockade is more effective than the respective monotherapies (table 2). High response rates and improvement of PFS and OS were already evident in 2 early phase trials investigating ipilimumab and nivolumab [24, 25]. However, the pivotal study for this drug combination was the 3 -armed phase III trial CheckMate 067 in which 945 treatment-naïve patients with unresectable or metastatic disease were randomized 1:1:1 to nivolumab plus ipilimumab, nivolumab alone, or ipilimumab alone, with PFS and OS as co-primary endpoints [26]. Data on the first parameter have already been published and revealed a response rate of $58 \%$ for the combination, $44 \%$ for nivolumab alone, and $19 \%$ for ipilimumab alone. Median PFS was 11.5, 6.9, and 2.9 months, respectively. The clinical benefit provided by the combination was most evident in patients with PD-L1-negative tumors. However, this high antitumor efficacy was accompanied by a high frequency of severe immune-related adverse events. In the combination cohort, virtually all patients experienced at least 1 side effect, while $57 \%$ showed an event of grade 3 or 4 . In $39 \%$ of all patients the combination therapy had to be stopped due to side effects such as diarrhea with colitis or hepatitis with elevated liver enzymes. However, no treatment-related death occurred in the combination arm of the study, and $85-100 \%$ of the severe adverse events were managed successfully and fully reversed with the appropriate therapeutic measures [26].

A post-hoc analysis on patients who discontinued ipilimumab and nivolumab due to severe adverse events in the phase II trial CheckMate 069 was recently presented [27]. After a minimum follow-up of 2 years, median PFS and OS were still not reached in randomized patients receiving ipilimumab and nivolumab and those who discontinued treatment. Thus, the treatment effects of this combination appeared durable with clinical benefit even for patients who discontinue therapy early [27].

\section{Ipilimumab plus Pembrolizumab}

The combination of ipilimumab and pembrolizumab has been assessed in the phase I/II trial KEYNOTE-029 for melanoma and renal cell carcinoma. Pembrolizumab was administered at $2 \mathrm{mg} / \mathrm{kg}$ body weight every 3 weeks with 4 cycles of ipilimumab at $1 \mathrm{mg} / \mathrm{kg}$ body weight. Recent data after a median follow-up of 10 months suggested that this combination was tolerable, as $72 \%$ of patients received all 4 doses of ipilimumab and no treatment-related deaths were observed. Severe immune-related adverse events were reported in $25 \%$ of patients. The best overall response rate was $57 \%$. However, no phase III trial data are available for pembrolizumab and ipilimumab to date [28].

\section{Triple Combination Therapy}

Combining tyrosine kinase inhibitors with immune checkpoint blockade may result in additional synergism. The triplet combination of BRAFi, MEKi, and anti-PD-1 showed high antitumor efficacy in preclinical studies. The phase I/II trial KEYNOTE-022 evaluates the combination of dabrafenib, trametinib and pembrolizumab in BRAFV600-mutated melanoma [29]. The dose-finding part demonstrated a manageable toxicity profile of the approved single-agent doses (dabrafenib $150 \mathrm{mg}$ BID, trametinib $2 \mathrm{mg}$ QID, pembrolizumab $2 \mathrm{mg} / \mathrm{kg}$ Q3W). 12 out of 13 patients (92.3\%) showed a decrease from baseline in the size of the target lesions. Phase II of this trial is currently further evaluating the safety and efficacy of this triplet combination compared to dabrafenib and trametinib plus placebo [29].

\section{Conclusion}

Patients with a BRAFV600 mutation should receive upfront BRAFi and MEKi as first- or second-line therapy. Currently, 2 combination regimens have been approved by the EMA: dabrafenib plus trametinib and vemurafenib plus cobimetinib. They have similar antitumor efficacy but a distinct profile of adverse events. For BRAF mutation-positive patients, the ideal sequence of targeted therapy with kinase inhibitors and immune checkpoint blockade is unclear and depends on the clinical course and individual patient characteristics.

Pembrolizumab and nivolumab show higher response rates compared to ipilimumab and fewer severe immune-related adverse events. However, the optimal treatment duration for these agents has not been defined yet and long-term data are still lacking. Unprecedented efficacy was recently yielded with combined CTLA-4 and PD-1 blockade, although more than half of all treated patients experienced an adverse event of grade 3 or 4 . However, no treatment-related deaths were recorded and the events could be adequately managed with close clinical monitoring early immunosuppression.

\section{Disclosure Statement}

M.V.H., C.H., S.A.G., and T.R. declare no conflict of interest. J.K.T. has received travel grants from BMS and speaker's honoraria from MSD, BMS, Roche and Novartis. C.B. has received speaker's fees and/or advisor's honoraria by Amgen, AstraZeneca, BMS, GSK, MSD, Novartis, Pierre Fabre, and Roche. 


\section{References}

1 Long GV, Stroyakovskiy D, Gogas H, Levchenko E, de Braud F, Larkin J, Garbe C, Jouary T, Hauschild A, Grob JJ, Chiarion Sileni V, Lebbe C, Mandala M, Millward M, Arance A, Bondarenko I, Haanen JB, Hansson J, Utikal J, Ferraresi V, Kovalenko N, Mohr P, Probachai V, Schadendorf D, Nathan P, Robert C, Ribas A, DeMarini DJ, Irani JG, Casey M, Ouellet D, Martin AM, Le N, Patel K, Flaherty K: Combined braf and mek inhibition versus braf inhibition alone in melanoma. N Engl J Med 2014;371:1877-1888.

2 Long GV, Stroyakovskiy D, Gogas H, Levchenko E, de Braud F, Larkin J, Garbe C, Jouary T, Hauschild A, Grob JJ, Chiarion-Sileni V, Lebbe C, Mandala M, Millward M, Arance A, Bondarenko I, Haanen JB, Hansson J, Utikal J, Ferraresi V, Kovalenko N, Mohr P, Probachai V, Schadendorf D, Nathan P, Robert C, Ribas A, DeMarini DJ, Irani JG, Swann S, Legos JJ, Jin F, Mookerjee B, Flaherty K: Dabrafenib and trametinib versus dabrafenib and placebo for val600 braf-mutant melanoma: A multicentre, double-blind, phase 3 randomised controlled trial. Lancet 2015;386:444-451.

3 Schadendorf D, Amonkar MM, Stroyakovskiy D, Levchenko E, Gogas H, de Braud F, Grob JJ, Bondarenko I, Garbe C, Lebbe C, Larkin J, Chiarion-Sileni V, Millward M, Arance A, Mandala M, Flaherty KT, Nathan P, Ribas A, Robert C, Casey M, DeMarini DJ, Irani JG, Aktan G, Long GV: Health-related quality of life impact in a randomised phase III study of the combination of dabrafenib and trametinib versus dabrafenib monotherapy in patients with BRAF V600 metastatic melanoma. Eur J Cancer 2015;51:833-840.

4 Dummer R, Schadendorf D, Ascierto PA, Arance Fernandez AM, Dutriaux C, Maio M, Rutkowski P, Del Vecchio M, Gutzmer R, Mandala M, Thomas L, Wasserman E, Ford J, Weill M, Sirulnik LA, Jehl V, Bozon V, Long GV, Flaherty K: Results of NEMO: A phase III trial of binimetinib (BINI) vs dacarbazine (DTIC) in NRAS-mutant cutaneous melanoma. ASCO Meeting Abstracts 2016;34:9500.

5 Robert C, Karaszewska B, Schachter J, Rutkowski P, Mackiewicz A, Stroiakovski D, Lichinitser M, Dummer R, Grange F, Mortier L, Chiarion-Sileni V, Drucis K, Krajsova I, Hauschild A, Lorigan P, Wolter P, Long GV, Flaherty K, Nathan P, Ribas A, Martin AM, Sun P, Crist W, Legos J, Rubin SD, Little SM, Schadendorf D: Improved overall survival in melanoma with combined dabrafenib and trametinib. N Engl J Med 2015;372: 30-39.

6 Grob JJ, Amonkar MM, Karaszewska B, Schachter J, Dummer R, Mackiewicz A, Stroyakovskiy D, Drucis K, Grange F, Chiarion-Sileni V, Rutkowski P, Lichinitser M, Levchenko E, Wolter P, Hauschild A, Long GV, Nathan P, Ribas A, Flaherty K, Sun P, Legos JJ, McDowell DO, Mookerjee B, Schadendorf D, Robert C: Comparison of dabrafenib and trametinib combination therapy with vemurafenib monotherapy on health-related quality of life in patients with unresectable or metastatic cutaneous BRAF Val600-mutation-positive melanoma (COMBI-v): Results of a phase 3, open-label, randomised trial. Lancet Oncol 2015;16:1389-1398.

7 Larkin J, Ascierto PA, Dreno B, Atkinson V, Liszkay G, Maio M, Mandala M, Demidov L, Stroyakovskiy D, Thomas L, de la Cruz-Merino L, Dutriaux C, Garbe C, Sovak MA, Chang I, Choong N, Hack SP, McArthur GA, Ribas A: Combined vemurafenib and cobimetinib in BRAF-mutated melanoma. N Engl J Med 2014;371: 1867-1876.

8 McArthur G, Larkin J, Dréno B, Ascierto P, Liszkay G, Maio M, Mandal' M, Demidov L, Stroyakovskiy D, Thomas L, De la Cruz-Merino L, Atkinson V, Dutriaux C, Garbe C, Wongchenko M, Hsu J, Koralek D,
Rooney I, Yan Y, Ribas A: Impact of baseline genetic heterogeneities on progression-free survival (PFS) in patients (pts) with advanced BRAFV600-mutated melanoma treated with cobimetinib (COBI) + vemurafenib (VEM) in the phase 3 coBRIM study. Eur J Cancer 2015;51:S722-S723.

9 Ribas A, Gonzalez R, Pavlick A, Hamid O, Gajewski TF, Daud A, Flaherty L, Logan T, Chmielowski B, Lewis K, Kee D, Boasberg P, Yin M, Chan I, Musib L, Choong N, Puzanov I, McArthur GA: Combination of vemurafenib and cobimetinib in patients with advanced BRAF(V600)-mutated melanoma: A phase $1 \mathrm{~b}$ study. Lancet Oncol 2014;15:954-965.

10 Pavlick AC, Ribas A, Gonzalez R, Hamid O, Gajewski T, Daud A, Puzanov I, Hsu JJ, Choong NW, McArthur GA: Extended follow-up results of phase Ib study (BRIM7) of vemurafenib (VEM) with cobimetinib (COBI) in BRAF-mutant melanoma. J Clin Oncol 2015;33:suppl; abstr 9020.

11 Jakob JA, Bassett RL Jr, Ng CS, Curry JL, Joseph RW, Alvarado GC, Rohlfs ML, Richard J, Gershenwald JE, Kim KB, Lazar AJ, Hwu P, Davies MA: NRAS mutation status is an independent prognostic factor in metastatic melanoma. Cancer 2012;118:4014-4023.

12 Yeh TC, Marsh V, Bernat BA, Ballard J, Colwell H, Evans RJ, Parry J, Smith D, Brandhuber BJ, Gross S, Marlow A, Hurley B, Lyssikatos J, Lee PA, Winkler JD, Koch K, Wallace E: Biological characterization of ARRY-142886 (AZD6244), a potent, highly selective mitogen-activated protein kinase kinase $1 / 2$ inhibitor. Clin Cancer Res 2007;13:1576-1583.

13 Solit DB, Garraway LA, Pratilas CA, Sawai A, Getz G, Basso A, Ye Q, Lobo JM, She Y, Osman I, Golub TR, Sebolt-Leopold J, Sellers WR, Rosen N: BRAF mutation predicts sensitivity to MEK inhibition. Nature 2006;439:358-362.

14 Ascierto PA, Schadendorf D, Berking C, Agarwala SS, van Herpen CM, Queirolo P, Blank CU, Hauschild A, Beck JT, St-Pierre A, Niazi F, Wandel S, Peters M, Zubel A, Dummer R: MEK162 for patients with advanced melanoma harbouring NRAS or Val600 BRAF mutations: A non-randomised, open-label phase 2 study. Lancet Oncol 2013;14:249-256.

15 Schadendorf D, Hodi FS, Robert C, Weber JS, Margolin K, Hamid O, Patt D, Chen TT, Berman DM, Wolchok JD: Pooled analysis of long-term survival data from phase II and phase III trials of ipilimumab in unresectable or metastatic melanoma. J Clin Oncol 2015; 33:1889-1894.

16 Daud A, Nandoskar P: Pembrolizumab for melanoma - safety profile and future trends. Expert Opin Drug Saf 2016:1-3.

17 Robert C, Ribas A, Wolchok JD, Hodi FS, Hamid O, Kefford R, Weber JS, Joshua AM, Hwu WJ, Gangadhar TC, Patnaik A, Dronca R, Zarour H, Joseph RW, Boasberg P, Chmielowski B, Mateus C, Postow MA, Gergich K, Elassaiss-Schaap J, Li XN, Iannone R, Ebbinghaus SW, Kang SP, Daud A: Anti-programmed-deathreceptor-1 treatment with pembrolizumab in ipilimumab-refractory advanced melanoma: A randomised dose-comparison cohort of a phase 1 trial. Lancet 2014;384:1109-1117.

18 Ribas A, Puzanov I, Dummer R, Schadendorf D, Hamid O, Robert C, Hodi FS, Schachter J, Pavlick AC, Lewis KD, Cranmer LD, Blank CU, O’Day SJ, Ascierto PA, Salama AK, Margolin KA, Loquai C, Eigentler TK, Gangadhar TC, Carlino MS, Agarwala SS, Moschos SJ, Sosman JA, Goldinger SM, Shapira-Frommer R, Gonzalez R, Kirkwood JM, Wolchok JD, Eggermont A, Li XN, Zhou W, Zernhelt AM, Lis J, Ebbinghaus S, Kang SP, Daud A: Pembrolizumab versus investigator- choice chemotherapy for ipilimumab-refractory melanoma (KEYNOTE-002): A randomised, controlled, phase 2 trial. Lancet Oncol 2015;16:908-918.

19 Robert C, Schachter J, Long GV, Arance A, Grob JJ, Mortier L, Daud A, Carlino MS, McNeil C, Lotem M, Larkin J, Lorigan P, Neyns B, Blank CU, Hamid O, Mateus C, Shapira-Frommer R, Kosh M, Zhou H, Ibrahim N, Ebbinghaus S, Ribas A: Pembrolizumab versus ipilimumab in advanced melanoma. $\mathrm{N}$ Engl J Med 2015;372:2521-2532.

20 Zimmer L, Goldinger SM, Hofmann L, Loquai C, Ugurel S, Thomas I, Schmidgen MI, Gutzmer R, Utikal JS, Goppner D, Hassel JC, Meier F, Tietze JK, Forschner A, Weishaupt C, Leverkus M, Wahl R, Dietrich U, Garbe C, Kirchberger MC, Eigentler T, Berking C, Gesierich A, Krackhardt AM, Schadendorf D, Schuler G, Dummer R, Heinzerling LM: Neurological, respiratory, musculoskeletal, cardiac and ocular side-effects of anti-PD-1 therapy. Eur J Cancer 2016;60:210-225.

21 Hofmann L, Forschner A, Loquai C, Goldinger SM, Zimmer L, Ugurel S, Schmidgen MI, Gutzmer R, Utikal JS, Goppner D, Hassel JC, Meier F, Tietze JK, Thomas I, Weishaupt C, Leverkus M, Wahl R, Dietrich U, Garbe C, Kirchberger MC, Eigentler T, Berking C, Gesierich A, Krackhardt AM, Schadendorf D, Schuler G, Dummer R, Heinzerling LM: Cutaneous, gastrointestinal, hepatic, endocrine, and renal side-effects of anti-PD-1 therapy. Eur J Cancer 2016;60:190-209.

22 Weber JS, D’Angelo SP, Minor D, Hodi FS, Gutzmer R, Neyns B, Hoeller C, Khushalani NI, Miller WH, Jr., Lao CD, Linette GP, Thomas L, Lorigan P, Grossmann KF, Hassel JC, Maio M, Sznol M, Ascierto PA, Mohr P, Chmielowski B, Bryce A, Svane IM, Grob JJ, Krackhardt AM, Horak C, Lambert A, Yang AS, Larkin J: Nivolumab versus chemotherapy in patients with advanced melanoma who progressed after anti-CTLA- 4 treatment (CheckMate 037): A randomised, controlled, open-label, phase 3 trial. Lancet Oncol 2015;16:375-384.

23 Robert C, Long GV, Brady B, Dutriaux C, Maio M, Mortier L, Hassel JC, Rutkowski P, McNeil C, KalinkaWarzocha E, Savage KJ, Hernberg MM, Lebbe C, Charles J, Mihalcioiu C, Chiarion-Sileni V, Mauch C, Cognetti F, Arance A, Schmidt H, Schadendorf D, Gogas H, Lundgren-Eriksson L, Horak C, Sharkey B, Waxman IM, Atkinson V, Ascierto PA: Nivolumab in previously untreated melanoma without BRAF mutation. N Engl J Med 2015;372:320-330.

24 Wolchok JD, Kluger H, Callahan MK, Postow MA, Rizvi NA, Lesokhin AM, Segal NH, Ariyan CE, Gordon RA, Reed K, Burke MM, Caldwell A, Kronenberg SA, Agunwamba BU, Zhang X, Lowy I, Inzunza HD, Feely W, Horak CE, Hong Q, Korman AJ, Wigginton JM, Gupta A, Sznol M: Nivolumab plus ipilimumab in advanced melanoma. N Engl J Med 2013;369:122-133.

25 Postow MA, Chesney J, Pavlick AC, Robert C, Grossmann K, McDermott D, Linette GP, Meyer N, Giguere JK, Agarwala SS, Shaheen M, Ernstoff MS, Minor D, Salama AK, Taylor M, Ott PA, Rollin LM, Horak C, Gagnier P, Wolchok JD, Hodi FS: Nivolumab and ipilimumab versus ipilimumab in untreated melanoma. N Engl J Med 2015;372:2006-2017.

26 Larkin J, Chiarion-Sileni V, Gonzalez R, Grob JJ, Cowey CL, Lao CD, Schadendorf D, Dummer R, Smylie M, Rutkowski P, Ferrucci PF, Hill A, Wagstaff J, Carlino MS, Haanen JB, Maio M, Marquez-Rodas I, McArthur GA, Ascierto PA, Long GV, Callahan MK, Postow MA, Grossmann K, Sznol M, Dreno B, Bastholt L, Yang A, Rollin LM, Horak C, Hodi FS, Wolchok JD: Combined nivolumab and ipilimumab or monotherapy in untreated melanoma. N Engl J Med 2015;373:23-34. 
27 Hodi FS, Postow MA, Chesney JA, Pavlick AC, Robert C, Grossmann KF, McDermott DF, Linette GP, Meyer N, Giguere JK, Agarwala S, Shaheen MF, Ernstoff MS, Minor DR, Salama AK, Taylor MH, Ott PA, Jiang J, Gagnier P, Wolchok JD: Overall survival in patients with advanced melanoma (MEL) who discontinued treatment with nivolumab (NIVO) plus ipilimumab (IPI) due to toxicity in a phase II trial (CheckMate 069). ASCO Meeting Abstracts 2016;34:9518.
28 Long GV, Atkinson V, Cebon JS, Jameson MB, Fitzharris BM, McNeil CM, Hill AG, Ribas A, Atkins MB, Thompson JA, Hwu W-J, Hodi FS, Menzies AM, Guminski AD, Kefford R, Shu X, Ebbinghaus S, Ibrahim N, Carlino MS: Pembrolizumab (pembro) plus ipilimumab (ipi) for advanced melanoma: Results of the KEYNOTE-029 expansion cohort. ASCO Meeting Abstracts 2016;34:9506.
29 Long GV, Hamid O, Hodi FS, Lawrence DP, Atkinson V, Starodub A, Carlino MS, Fisher RA, Miller WH, Maio M, Butler M, Queirolo P, Ferrucci PF, Petrella TM, Schachter J, Huang Y, Diede SJ, Ebbinghaus S, Ribas A: Phase 2 study of the safety and efficacy of pembrolizumab (pembro) in combination with dabrafenib (D) and trametinib (T) for advanced melanoma (KEYNOTE-022). ASCO Meeting Abstracts 2016;34:TPS9596. 